(C) 1984. The Genetical Society of Great Britain

\title{
POLYGENE POLYMORPHISM AFFECTING WING VEIN FORMATION \\ IN A NATURAL POPULATION OF DROSOPHILA MELANOGASTER
}

\author{
J. M. THODAY* AND JAMES N. THOMPSON, JR. $\dagger$ \\ * Department of Genetics, University of Cambridge, Cambridge, England; † Department \\ of Zoology, University of Oklahoma, Norman, Oklahoma, USA
}

Received 31.i.84

\section{SUMMARY}

One hundred isofemale lines originating from the same (Oklahoma) wild population of Drosophila melanogaster were assayed for cryptic variation capable of producing gaps in wing vein L5 in flies heterozygous for genomes from an enhanced veinlet line.

There were very significant differences between isofemale lines, and the Oklahoma population was clearly polymorphic for vein-gap genes of subthreshold expression. Many of the isofemale lines were also segregating for such genes.

There were no obvious relationships between this polymorphism and others that have been studied in the Oklahoma lines.

\section{INTRODUCTION}

Natural population gene pools are segregating for many polygenic loci that affect morphological traits, though the loci involved in such quantitative traits are difficult to identify and their relationship to other polymorphisms is often unclear. In an intensive study of modifiers of wing vein phenotype in Drosophila melanogaster, we showed that a veinlet (ve) line selected for extremely short veins often gave $F_{1}$ flies with a high frequency of vein gaps when crossed to wild-type stocks (Thompson and Thoday, 1972). The polygenic "modifiers" accumulated in the ve selection line are not specific to veinlet expression. They have comparable effects upon other venation mutants, such as cubitus interruptus, radius incompletus, plexus, and net, and must therefore be loci that influence processes basic to the establishment of the wing vein pattern.

In a genetic assimilation experiment similar to those carried out by Waddington (1953) and others, we found that some homozygous combinations of ve modifiers are capable of producing vein gaps even in the absence of veinlet, though in normal combinations they are sub-threshold in effect (Thompson and Thoday, 1975). The availability of this sensitive veinlet tester stock led us to survey a series of isofemale lines from a single natural population in order to measure the extent to which the population is segregating for polygenic modifiers of vein formation.

To this end, over 100 separate females were collected around a single bait in Oklahoma and were used to establish 100 replicated isofemale lines. These were used to screen for polygenic modifiers of vein formation in order to estimate their heterogeneity in a natural population. In addition, these strains allowed us to compare the amount of polygenic variation with 
the level of segregation for other genetic components, such as electrophoretic alleles and chromosomal re-arrangements.

\section{Materials and Methods}

A set of 100 pairs of isofemale strains was established from females collected around a watermelon bait on a single afternoon in late September 1973. After a quarantine period for mites, one of each pair was tested for modifiers of vein development. Most lines were screened between January and April 1974, but a few were tested later. Although assays were carried out over a period of time, lines were mass-transferred to minimise any inbreeding while in culture.

Each line was screened for polygenic modifiers of vein formation that caused terminal gaps to appear in the fifth longitudinal (L5) wing vein in flies heterozygous for chromosomes from the veinlet Short selection line (ve Short-IV; Thompson, 1974). A typical L5 gap is shown in plate 1. Crosses were carried out by mating ten single males from a given isofemale line to virgins from ve Short. From each $F_{1}$ culture, 20 females and 20 males were scored for L5 vein gaps in the right-hand wing. Focusing upon the right wing was done to avoid sampling bias, though we found that the expression of gaps was generally similar in the two wings.

All lines and crosses were maintained at $25 \pm 1{ }^{\circ} \mathrm{C}$ on a standard oatmeal, molasses, agar medium seeded with a suspension of live yeast. Mutations are described in Lindsley and Grell (1967).

\section{RESUlts}

The total number of wings with gaps is given for males and females in table 1 ( $N=200$ wings of each sex scored per strain). A comparison of overall gap frequencies enables us to evaluate heterogeneity between isofemale strains. Table 2 presents the results of an analysis of the heterogeneity shown for all 1,000 progenies ( 10 crosses $\times 100$ strains). It is clear that the lines differ greatly with respect to genes that lead to L5 gaps in the sensitive ve Short background. The original Oklahoma population must have been highly polymorphic at relevant loci.

This being established it is of interest to inquire how many of the isofemale lines themselves were still polymorphic when tested. The heterogeneity $\chi_{(9)}^{2}$ for each line is given in table 1 . These were calculated as $G$ (Sokal and Rohlf, 1969) because gap frequencies in many of the lines are too low for conventional $\chi^{2}$ estimates to be accurate. A total of 81 of the 100 lines show significant heterogeneity. Thus, the ten progenies produced by different males tested for the same isofemale line are significantly different in about 80 per cent of the lines.

The very high heterogeneity $\chi_{(G)}^{2}$ values for many lines clearly indicate that these lines are still segregating at relevant polygenic loci. Some part of the overall heterogeneity might, however, arise from two other potential sources-environmental differences between cultures or residual heterozygosity of the ve Short tester line.

Environmental differences are expected to play some part in producing the observed heterogeneity among crosses, since vein formation, like essentially all quantiative traits, is sensitive to environmental influences such as 


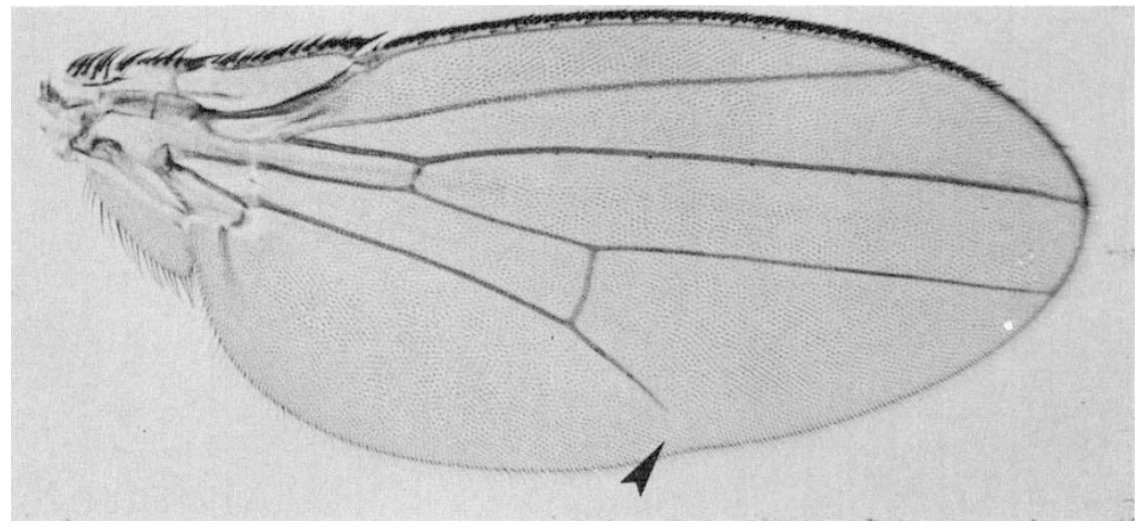

Plate. Wing of D. melanogaster heterozygous for ve short. The terminal LS vein gap is indicated by an arrow. 
TABLE 1

The number of females and males with $L 5$ vein gaps among the ten $F_{1}$ progenies (of $20 \% 9$ and 2080 each) from each of the $100 \mathrm{OK}$ lines and the heterogeneity between the ten progenies

\begin{tabular}{|c|c|c|c|c|c|c|c|}
\hline Line & 우 & $\delta$ & $\chi^{2}(G)$ & Line & $q$ & 0 & $\chi^{2}(G)$ \\
\hline 1 & 60 & 53 & $70 \cdot 27$ & 51 & 18 & 19 & $105 \cdot 97$ \\
\hline 2 & 26 & 11 & $48 \cdot 60$ & 2 & 42 & 29 & $84 \cdot 64$ \\
\hline 3 & 3 & 2 & $(12 \cdot 64)$ & 3 & 0 & 1 & $(4 \cdot 63)$ \\
\hline 4 & 4 & 2 & $19 \cdot 19$ & 4 & 19 & 30 & 55.06 \\
\hline 5 & 1 & 1 & $(9 \cdot 30)$ & 5 & 13 & 17 & $35 \cdot 75$ \\
\hline 6 & 99 & 92 & $60 \cdot 33$ & 6 & 31 & 24 & $149 \cdot 68$ \\
\hline 7 & 6 & 3 & $25 \cdot 29$ & $7^{*}$ & 22 & 10 & $60 \cdot 27$ \\
\hline 8 & 0 & 0 & - & 8 & 12 & 7 & $26 \cdot 91$ \\
\hline $9^{* *}$ & 27 & 9 & $44 \cdot 39$ & 9 & 31 & 39 & $81 \cdot 21$ \\
\hline 10 & 25 & 15 & $69 \cdot 88$ & 60 & 125 & 132 & $109 \cdot 15$ \\
\hline 11 & 43 & 40 & $122 \cdot 49$ & 1 & 74 & 58 & $123 \cdot 76$ \\
\hline 12 & 60 & 63 & $84 \cdot 53$ & 2 & 15 & 14 & $78 \cdot 81$ \\
\hline 13 & 24 & 19 & $53 \cdot 87$ & 3 & 2 & 0 & $(9 \cdot 30)$ \\
\hline 14 & 0 & 3 & $(7 \cdot 28)$ & 4 & 13 & 10 & 35.90 \\
\hline $15^{* *}$ & 81 & 40 & $44 \cdot 34$ & 5 & 3 & 0 & $(10 \cdot 10)$ \\
\hline 16 & 39 & 36 & $103 \cdot 27$ & 6 & 17 & 9 & $39 \cdot 25$ \\
\hline 17 & 15 & 21 & $39 \cdot 88$ & 7 & 74 & 57 & 155.49 \\
\hline 18 & 10 & 8 & $49 \cdot 82$ & 8 & 22 & 24 & $98 \cdot 55$ \\
\hline 19 & 40 & 52 & $65 \cdot 66$ & 9 & 10 & 8 & $50 \cdot 30$ \\
\hline 20 & 0 & 2 & $(6 \cdot 48)$ & 70 & 9 & 14 & $(10.91)$ \\
\hline 21 & 10 & 11 & 52.09 & $1^{*}$ & 19 & 9 & 38.65 \\
\hline 22 & 30 & 23 & 48.02 & 2 & 22 & 40 & $18 \cdot 54$ \\
\hline 23 & 0 & 0 & - & 3 & 7 & 8 & $34 \cdot 33$ \\
\hline 24 & 69 & 67 & $53 \cdot 22$ & 4 & 1 & 0 & $(4 \cdot 63)$ \\
\hline $25^{*}$ & 25 & 42 & $171 \cdot 85$ & 5 & 1 & 1 & $(6.48)$ \\
\hline 26 & 42 & 43 & $105 \cdot 40$ & 6 & 58 & 69 & 140.87 \\
\hline 27 & 19 & 12 & 63.97 & $7^{* * *}$ & 1 & 12 & $26 \cdot 78$ \\
\hline 28 & 32 & 38 & $97 \cdot 45$ & $8^{*}$ & 39 & 58 & $77 \cdot 19$ \\
\hline 29 & 11 & 10 & $24 \cdot 34$ & 9 & 12 & 8 & $19 \cdot 27$ \\
\hline 30 & 26 & 11 & $54 \cdot 00$ & 80 & 23 & 28 & 119.83 \\
\hline 31 & 25 & 25 & 42.92 & 1 & 118 & 111 & $119 \cdot 15$ \\
\hline 32 & 9 & 8 & $20 \cdot 27$ & $2^{* *}$ & 47 & 15 & $30 \cdot 39$ \\
\hline 33 & 13 & 8 & $53 \cdot 52$ & 3 & 0 & 0 & - \\
\hline 34 & 41 & 36 & 117.96 & 4 & 12 & 10 & $76 \cdot 53$ \\
\hline 35 & 16 & 9 & $34 \cdot 14$ & 5 & 20 & 28 & 78.04 \\
\hline 36 & 4 & 1 & $(16 \cdot 57)$ & $6^{*}$ & 16 & 6 & $36 \cdot 26$ \\
\hline 37 & 76 & 83 & $147 \cdot 15$ & 7 & 51 & 49 & $92 \cdot 25$ \\
\hline 38 & 49 & 46 & 196.44 & 8 & 13 & 10 & $62 \cdot 86$ \\
\hline 39 & 25 & 23 & $50 \cdot 21$ & 9 & 39 & 44 & 150.09 \\
\hline 40 & 25 & 32 & $64 \cdot 51$ & 90 & 21 & 15 & $132 \cdot 13$ \\
\hline 41 & 71 & 61 & $163 \cdot 87$ & 1 & 0 & 3 & $(7 \cdot 28)$ \\
\hline 42 & 9 & 9 & $40 \cdot 50$ & 2 & 16 & 18 & 115.00 \\
\hline 43 & 1 & 0 & $(4 \cdot 63)$ & $3^{*}$ & 26 & 14 & 89.69 \\
\hline 44 & 26 & 35 & $44 \cdot 79$ & 4 & 29 & 17 & $99 \cdot 80$ \\
\hline 45 & 4 & 6 & $26 \cdot 19$ & 5 & 63 & 51 & $226 \cdot 26$ \\
\hline 46 & 10 & 8 & $20 \cdot 64$ & 6 & 4 & 0 & $(7 \cdot 39)$ \\
\hline 47 & 19 & 19 & $63 \cdot 35$ & 7 & 32 & 33 & $141 \cdot 71$ \\
\hline 48 & 42 & 35 & 62.94 & 8 & 0 & 0 & - \\
\hline 49 & 3 & 6 & $(16 \cdot 15)$ & $9^{*}$ & 49 & 30 & 140.66 \\
\hline 50 & 68 & 69 & $166 \cdot 62$ & 100 & 4 & 4 & $17 \cdot 84$ \\
\hline
\end{tabular}

$\chi^{2}=16 \cdot 92,21 \cdot 67,27 \cdot 88$.

$P=0.05,0 \cdot 01,0.001$.

Significant sex differences are asterisked. ${ }^{*} p<0.05,{ }^{* *} p<0.01$. Line 72 has significant $(p<0.01)$ sex $\times$ progeny interaction. 
TABLE 2

Between and within line heterogeneity of gap frequencies

\begin{tabular}{lcccccc}
\hline Source & $x^{2}(\mathrm{G})$ & $\mathrm{N}$ & $\mathrm{P}$ & $\mathrm{MS}$ & $\mathrm{F}$ & $\mathrm{P}$ \\
\hline $\begin{array}{l}\text { Between lines } \\
\begin{array}{l}\text { Within lines between } \\
\text { progenies }\end{array}\end{array}$ & 3531.3320 & 99 & Small & 35.6700 & 3.9475 & Small \\
\hline Total & 8132.5148 & 900 & Small & 9.0361 & - & \\
\hline
\end{tabular}

temperature shifts during development. We carefully controlled culture conditions to minimise non-genetic sources of variation, but environmental differences are almost impossible to separate with confidence from the effects of any residual heterozygosity in the selected ve Short tester line.

However, we can estimate the overall effects of all extraneous sources of variation by assaying gap frequencies in replicate crosses of ve Short to the highly inbred Oregon wild type strain. The ve Short line was crossed to Oregon as part of a separate series of experiments at generations S7 and S35 of selection. At S7, five Oregon males were separately tested, and the results were heterogeneous $\left(G_{(4)}=16.60, p<0.01\right)$. At S35 only three Oregon males were tested $\left(G_{(2)}=5.87,0.05>p>0.01\right)$. Combining these samples, $\chi_{(7)}^{2}=24.12$, which gives a mean square of 3.45 or $a \chi_{(9)}^{2}$ of 31.02 . The $F_{9 / 9}$ for significance at the $p \simeq 0.05$ level is $3 \cdot 18$, which would indicate that any $\chi^{2}$ greater than 98.64 in table 1 is not likely to have arisen from heterogeneity in the ve Short tester or environmental factors alone. On this test 23 of the lines are segregating for polygenic modifiers of vein formation.

We believe that this is a very conservative estimate, since it is based upon tests of $v e$ Short before it had finished responding to selection. If we consider only the limited data for $\mathrm{S} 35$, the $28 \chi^{2}$ values above 83.96 would indicate genetic differences among the males of a line. The estimate is still conservative, since it assumes that environmental effects are negligible, the Oregon stock is genetically uniform, and the ve Short line did not become more uniform in spite of continued selection and inbreeding after these Oregon tests had been conducted.

Another way to evaluate the level of heterogeneity within a line involves variance ratios for the data in table 1 . Assume that the smallest $G_{(9)}$ that is significant is 20.64 (for line 46 ; line 72 is ignored here as discussed below). Then any line having a $G$ of $20.64 \times 3 \cdot 18=65.64$ has significantly greater heterogeneity than line 46 . A total of 36 lines are included within this category. Although each of these arguments is indirect, we regard the evidence as supporting the idea that at least a quarter of the lines were still segregating for polygenic loci when tested.

Line 72 shows a different kind of variation. Table 3 gives the gap frequencies for all ten replicate crosses and an analysis of $\chi_{(G)}^{2}$. There is not only a significant overall sex difference, but the sex $\times$ progenies item is significant which shows that line 72 was segregating for $X$ chromosomes of different effect.

There are other significant sex differences, though none involve a sex $x$ progenies interaction. Eight lines had significantly more females with gaps 
TABLE 3

Data for line 72

\begin{tabular}{ccccc}
\hline & \multicolumn{2}{c}{$\%$} & \multicolumn{2}{c}{$\delta$} \\
\cline { 2 - 5 } Progeny of $\delta$ & Gaps & No gaps & Gaps & No gaps \\
\hline 1 & 0 & 20 & 7 & 13 \\
2 & 2 & 18 & 5 & 15 \\
3 & 5 & 15 & 6 & 14 \\
4 & 4 & 16 & 1 & 19 \\
5 & 1 & 19 & 4 & 16 \\
6 & 6 & 14 & 5 & 15 \\
7 & 0 & 20 & 1 & 19 \\
8 & 0 & 20 & 3 & 17 \\
9 & 3 & 17 & 2 & 18 \\
10 & 1 & 19 & 6 & 14 \\
\hline Total & 22 & 278 & 40 & 260 \\
\hline
\end{tabular}

Analysis of $\chi^{2}(G)$

\begin{tabular}{lrrc} 
Source & G & N & P \\
\hline Progenies & 18.5383 & 9 & $<0.05$ \\
Sex & 6.2608 & 1 & $<0.02$ \\
Interaction & 21.9291 & 9 & $<0.01$
\end{tabular}

than males, indicating that their $X$ chromosomes might be carrying recessive loci that reduced gap penetrance. These eight lines are homogeneous in this respect, so their $X$ chromosomes could all be identical. In these lines the average frequency of females with gaps is 18 per cent, while only 8 per cent of the males have L5 gaps.

In addition, there are two other lines ( 25 and 77 ) in which significantly more male than female wings have gaps. These lines could be homozygous for one of the $X$ chromosomes that segregated in line 72 . When we remove those lines that show significant sex differences, we are left with only a small difference between the sexes (13.15 per cent of the females and $12 \cdot 30$ per cent of the males have L5 gaps), though it is still significant $\left(\chi_{(1)}^{2}(\mathrm{G})=\right.$ $5.64, p<0.02$ ). This may be due to the summation of lines that have the presumptive recessive $X$-linked polygenic loci that influences gap frequency, but that are not marked in table 1 because the sex difference is not significant in individual samples. Although there may also be some sex-limited effect in some lines, this is less likely because the reduction of vein formation is generally more extreme in males (Thompson, 1974).

One further aspect of the results should be mentioned. The data discussed above concern only gaps in the L5 vein. However, 29 lines yielded flies with gaps in others veins, particularly L4. Not all of these had gaps in the L5 vein. Line 94, for example, had one fly with gaps in the L2, L3, and L5. Line 90 clearly differed from all others; 8 of the scored progenies had no gaps, while one gave 12 females and 7 males with L5 gaps, and the other gave 9 females and 8 males with large gaps in the L2, L4, and L5. It is possible that line 90 is segregating for an isoallele of veinlet or some other major vein mutation, although a rare combination of polygenic modifiers 
could theoretically produce the same effect. Thus, we may conclude that several polygenic factors are segregating in the population, though apparently not at similar frequencies.

\section{Discussion}

The 100 isofemale lines established from a single collection in Oklahoma differed significantly with respect to genes that, in heterozygous combination with the ve Short genotype, led to defective formation of the L5 and other wing veins. Furthermore, some lines differed in $X$-linked polygenic loci. and there is suggestive evidence that many of the individual isofemale lines were autosomally heterogeneous.

The Oklahoma population was, therefore, highly polymorphic for polygenic modifiers of wing venation even though none of the "wild type" flies collected from nature have gaps in their veins. The polymorphism is cryptic and is only revealed by crosses to a tester stock that alters the threshold for expression of the defects.

We do not speculate here about the maintenance of this polymorphism, though it could be selectively maintained or neutral, depending upon the other segregating loci and conditions during development. One would predict, however, that selection could not significantly affect polygene allele frequencies once the penetrance of vein gaps had been reduced to zero, unless the polygenes have pleiotropic effects. No major pleiotropic effects have been detected in studies of other vein formation polygenes (Thompson, 1974, 1979).

The availability of these lines also gave us an opportunity to ask whether the polymorphism revealed for vein formation shows any relationship to other polymorphisms that have been studied in these Oklahoma isofemale lines. Downes (1982) and Downes and Thoday (1982), for example, measured variation in electrophoretic mobility of 14 enzymes in replicates ( $a$ and $b$ ) of isofemale lines 1 to 10 . Nine enzymes were heterozygous in at least one of the 20 lines (the overall frequency of the Fast-mobility allele is given in parentheses): $\alpha$ Gpdh (0.96), Acph-1 (0.67), Adh (0.38), Est-C (3 alleles; 0.99), Est-6 (0.38), Fk (0.24), Odh (0.93), Pgm (0.91), and $Z w$ $(0 \cdot 10)$. No variation was detected for $A l d, M d h-1, M d h$-NAPD, $1 d h$-NAPD, and $X d h$.

Inversion 3R Payne occurs in seven pairs of lines with an overall frequency of $0 \cdot 28$, and three other inversions occur at relatively low frequencies. There is no evidence that gap frequencies are correlated with either inversion types or specific electrophoretic alleles.

Isofemale lines 1 to 10 also vary for sternopleural chaeta number (table 4). Sirce gap frequencies were taken before the replicate cultures of lines 1 to 10 were separately labelled ( $1 \mathrm{a}, 1 \mathrm{~b}$, and so forth), we have estimated the correlation between gap frequencies and the average of the $a$ and $b$ culture chaeta numbers $(r=-0 \cdot 2$, not significant). These lines also vary greatly in both the $X$ and $Y$ rDNA (Coen, Thoday, and Dover, 1982). Again, there is no significant corelation between these polymorphisms.

Finally, the Oklahoma lines carry mutator factors of the hybrid dysgenesis sytem (Thompson and Woodruff, 1978; Woodruff and Thompson, 1980). Woodruff and Thompson (1977) tested lines 1 to 25 and found all to induce male recombination in the progeny of outcrosses to the sensitive 
TABLE 4

Data from P. Kearns (unpublished)

\begin{tabular}{|c|c|c|c|c|c|c|c|c|c|c|}
\hline \multirow{3}{*}{$\begin{array}{l}\text { Sub } \\
\text { Line }\end{array}$} & \multicolumn{10}{|c|}{ Mean sternopleural chaeta number in $O K$ lines $1 \mathrm{a}-10 \mathrm{~b}$} \\
\hline & & & & & & ine & & & & \\
\hline & 1 & 2 & 3 & 4 & 5 & 6 & 7 & 8 & 9 & 10 \\
\hline a & 15 & 16 & 18 & 16 & 17 & 15 & 17 & 17 & $14 \cdot 4$ & 13.9 \\
\hline b & $15 \cdot 1$ & 14.6 & $17 \cdot 4$ & $17 \cdot 8$ & $16 \cdot 0$ & 18.0 & 17.6 & $17 \cdot 6$ & 14.9 & $14 \cdot 3$ \\
\hline
\end{tabular}

Analysis of variance

\begin{tabular}{|c|c|c|c|c|c|c|c|}
\hline Source & SS & & $\mathbf{N}$ & & MS & F & $\mathbf{P}$ \\
\hline Sex & $45 \cdot 56$ & & 1 & & & $20 \cdot 8$ & small \\
\hline Between line pairs & & $632 \cdot 36$ & & 9 & $70 \cdot 26$ & $32 \cdot 1$ & small \\
\hline Within line pairs & & $140 \cdot 23$ & & 10 & $14 \cdot 02$ & $6 \cdot 4$ & small \\
\hline Total lines & $772 \cdot 59$ & & 19 & & 40.66 & $18 \cdot 6$ & small \\
\hline Lines $\times \operatorname{sex}$ & $35 \cdot 28$ & & 19 & & 1.86 & - & \\
\hline Error (individuals) & $778 \cdot 50$ & & 360 & & $2 \cdot 19$ & & \\
\hline Total & $1641 \cdot 93$ & & 399 & & & & \\
\hline
\end{tabular}

$d p b c n b w$ tester strain. The amount of male recombination is heterogeneous in their data $\left(G_{20}=291.83, p<0.001\right)$, but there is no significant correlation with gap frequency $(r=0 \cdot 149$, with 25 pairs $)$.

In summary, we have found that an extensive sample of genomes from a natural population is highly polymorphic for cryptic polygenic loci and the maintenance of this polymorphism can not be explained by its association with sampled inversions, enzyme polymorphism, chaeta modifiers or rDNA genes. Furthermore, the formation of gaps is not a somatic expression of a developmental instability induced by hybrid dysgenesis. Milkman (see 1970: Boyer et al., 1973) also found extensive cryptic polygenic segregations affecting vein formation, and many other quantifiable traits show similar modifiability (e.g., Thompson and Spivey, 1984).

It is striking that natural populations carry such high frequencies of polygenic modifiers for phenotypes that do not normally vary in natural populations. An increased understanding of the extent of such genetic variation should help contribute to our appreciation of the potential variability provided by the genetic structure of natural populations.

Acknowledgements. This work was supported in part by a Science Research Council grant to J.M.T. and National Science Foundation Grant No. BSR-8300025 to J.N.T.

\section{REFERENCES}

BOYER, B. J., PARRIS, D. L. AND MILKMAN, R. 1973. The crossveinless polygenes in an Iowa population. Genetics, 75, 169-179.

COEN, E. S., THODAY, J. M. AND DOVER, G. 1982. Rate of turnover of structural variants in the rDNA gene family of Drosophila melanogaster. Nature, 295, 564-568.

DOWNES, S. P. 1982. Studies on populations of Drosophila melanogaster in homogeneous and heterogeneous environments. PhD. Thesis, University of Cambridge.

DOWNES, S. P. AND THODAY, J. M. 1982. Effect of yeasts on polymorphism at the Adh locus in Drosophila melanogaster. Heredity, 49, 221-234. 
LINDSLEY, D. L. AND GRELL, E. H. 1967. Genetic Variations of Drosophila melanogaster. Carnegie Institution of Washington, 627.

MILKMAN, R. 1970. The genetic basis of natural variation in Drosophila melanogaster. Adv. in Genet., 15, 55-114.

SOKAL, R. R. AND ROHLF, F. J. 1969. Biometry. W. H. Freeman, San Francisco.

THOMPSON, J. N., JR. 1974. Studies on the nature and function of polygenic loci in Drosophila. I. Comparison of genomes from selection lines. Heredity, 33, 373-387.

THOMPSON, J. N., JR. 1979. Polygenic influences upon development in a model character. In Thompson, J. N. and Thoday, J. M. (eds.), Quantitative Genetic Variation, Academic Press, New York, pp. 243-261.

THOMPSON, J. N., JR. AND SPIVEY, W. E. 1984. Organization of polygenic systems: Cell death modifiers from natural populations of Drosophila melanogaster. (submitted).

THOMPSON, J. N., JR. AND THODAY, J. M. 1972. Modification of dominance by selection in the homozygote. Heredity, 29, 285-292.

THOMPSON, J. N., JR. AND THODAY, J. M. 1975. Genetic assimilation of part of a mutant phenotype. Genet. Res., Camb., 26, 149-162.

THOMPSON, J. N., JR. AND WOODRUFF, R. C. 1978. Mutator genes-pacemakers of evolution. Nature, 274, 317-321.

WADDINGTON, C. H. 1953. Genetic assimilation of an acquired character. Evolution, 7, 118-126. WOODRUFF, R. C. AND THOMPSON, J. N. JR. 1977. An analysis of spontaneous recombination in Drosophila melanogaster males: Isolation and characterisation of male recombination lines. Heredity, 38, 291-307.

WOODRUFF, R. C. AND THOMPSON, J. N. JR, 1980. Hybrid release of mutator activity and the genetic structure of natural populations. Evolutionary Biol, 12, 129-162. 